\title{
Subdivision of Nasopharyngeal Carcinoma Patients with Bone-Only Metastasis at Diagnosis for Prediction of Survival and Treatment Guidance
}

\author{
Xue-Song Sun, MD ${ }^{1,2}$ \\ Yu-Jing Liang, MD',2 \\ Sai-Lan Liu, MD1,2 \\ Qiu-Yan Chen, PhD',2 \\ Shan-Shan Guo, $\mathrm{PhD}^{1,2}$ \\ Yue-Feng Wen, $\mathrm{PhD}^{1,2}$ \\ Li-Ting Liu, $\mathrm{PhD}^{1,2}$ \\ Hao-Jun Xie, MD ${ }^{1,2}$ \\ Qing-Nan Tang, $\mathrm{MD}^{1,2}$ \\ Xiao-Yun Li, MD1,2 \\ Jin-Jie Yan, MD',2 \\ Lin-Quan Tang, $\mathrm{PhD}^{1,2}$ \\ Hai-Qiang Mai, $P h D^{1,2}$
}

${ }^{1}$ Sun Yat-sen University Cancer Center, State Key Laboratory of Oncology in South China, Collaborative Innovation Center for Cancer Medicine, Guangzhou, ${ }^{2}$ Department of Nasopharyngeal Carcinoma, Sun Yat-sen University Cancer Center, Guangzhou, China

Correspondence: Hai-Qiang Mai, MD, PhD Department of Nasopharyngeal Carcinoma, Sun Yat-sen University Cancer Center, 651 Dongfeng Road East,

Guangzhou 510060, China

Tel: 86-20-87343643

Fax: 86-20-87343392

E-mail: maihq@mail.sysu.edu.cn

Co-correspondence: Lin-Quan Tang, MD, PhD Department of Nasopharyngeal Carcinoma, Sun Yat-sen University Cancer Center, 651 Dongfeng Road East,

Guangzhou 510060, China

Tel: 86-20-87343643

Fax: 86-20-87343392

E-mail: tanglq@sysucc.org.cn

Received November 27, 2018

Accepted January 2, 2019

Published Online January 4, 2019

*Xue-Song Sun, Yu-Jing Liang, and Sai-Lan Liu contributed equally to this work.

\section{Purpose}

The purpose of this study was to subdivide M1 stage nasopharyngeal carcinoma (NPC) patients with bone-only metastases for prognosis prediction while identifying the treatment effect of locoregional radiotherapy (LRRT) and metastasis radiotherapy (MRT) among patients with different risk.

\section{Materials and Methods}

From November 2006 to October 2016, a total of 226 patients with bone-only metastasic NPC were retrospectively enrolled. All patients developed distant lesions before receiving treatment. All potential prognostic factors were considered and the correlation of the M1 subdivisions with overall survival (OS) was determined by Cox regression hazards model. Kaplan-Meier curves were used to appraise survival condition and log-rank testing was used to compare the differences.

\section{Results}

The median follow-up time was 33.9 months (range, 3 to 126 months). According to multivariate Cox proportional hazard analysis, the number of metastatic lesions and Epstein-Barr virus (EBV) DNA status after palliative chemotherapy (PCT) were independent prognostic factors for OS. Thus, we subdivided patients into three risk groups according to these two factors. Systemic chemotherapy combined with LRRT may benefit patients in low- and intermediate-risk groups but not in the high-risk group. Further aggressive MRT based on systemic chemotherapy showed no survival benefit in any risk group.

\section{Conclusion}

The stratification of NPC patients with bone-only metastasis based on EBV DNA after PCT and the number of metastatic lesions provided promising prognostic value and could aid clinicians in person-specific treatment.

\author{
Key words \\ Nasopharyngeal carcinoma, Metastasis, Epstein-Barr virus, \\ Radiotherapy, Survival
}




\section{Introduction}

Nasopharyngeal carcinoma (NPC) is a malignancy considered to be epidemic in east and Southeast Asia, especially in Southern China. Indeed, it has been notoriously described as Canton Tumor. There were 86,700 new cases of NPC and 50,800 deaths reported worldwide in 2012 [1]. NPC is distinguished from other head and neck carcinomas by geographic distribution, where Epstein-Barr virus infection is a major precipitant and has great tendency to result in distant metastases [2]. Due to the concealed location of the nasopharynx, many patients tend to be at an advanced stage of disease at initial diagnosis. A previous study reported that approximately 15\% NPC patients were diagnosed as having metastasis before any treatment [3]. Survival times vary widely in NPC patients with distant metastasis at initial diagnosis [4,5]. However, the TNM stage, which is widely used to aid clinicians in prognosis prediction, has shown no value in staging patients diagnosed as having M1 stage. Thus, stratifying the patients with metastatic NPC is necessary for clinicians to classify patients and guide appropriate treatment [6].

Bone metastasis is the most frequently metastatic site, with an estimated incidence of $54 \%-80 \%$ [7-9]. Thus, there is a need for a prognostic model to predict survival risk in those with metastasis. Shen et al. [5] divided bone metastatic NPC into three risk groups using spine involvement factors and metastatic sites number. Similarly, Chen et al. [10] estab- lished a prognostic score using six factors to discriminate patients into low-/high-risk groups. However, both studies involved patients who developed metastasis after initial treatment. Besides, Epstein-Barr virus (EBV) DNA, which is an important biomarker for NPC, was not included in the analysis [11]. Platinum-based combination chemotherapy is recommended for metastatic NPC patients according to the National Comprehensive Cancer Network (NCCN) guidelines [12]. However, there is no standard model which considers whether radiotherapy should be applied to patients with primary head and neck tumors with distant metastases. Studies focusing on the application of ablative treatment to the metastasis site are rare.

Therefore, the purpose of our study was to divide patients with bone-only metastasis into different risk groups. Furthermore, we aimed to investigate whether these patients could benefit from locoregional radiotherapy (LRRT) and metastasis radiotherapy (MRT) in the different risk groups.

\section{Materials and Methods}

\section{Patients}

Between November 2006 and October 2016, 226 consecutive newly diagnosed bone-only metastatic NPC patients in

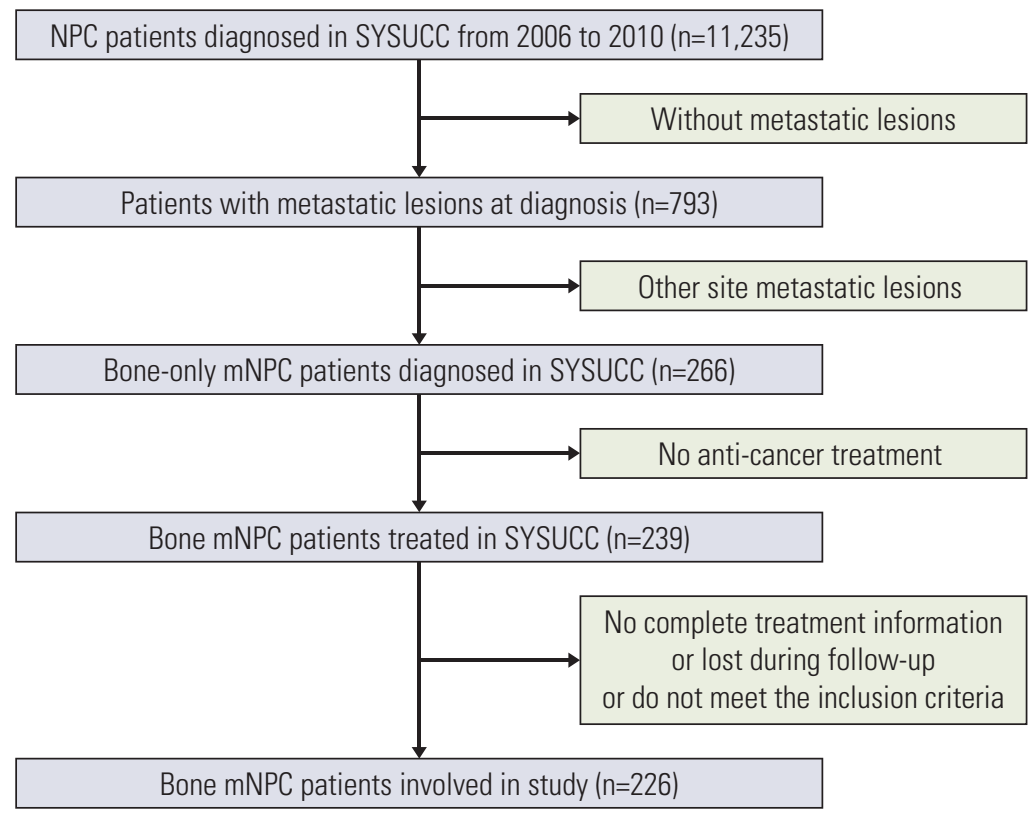

Fig. 1. Flow chart for patient inclusion. NPC, nasopharyngeal carcinoma; SYSUCC, Sun Yat-sen University Cancer Center; $\mathrm{mNPC}$, metastatic nasopharyngeal carcinoma. 
Sun Yat-sen University Cancer Center (SYSUCC), China, were enrolled in our analysis. The inclusion criteria were as follows: (1) pathological diagnosis of NPC; (2) cisplatinbased palliative chemotherapy (PCT); (3) Karnofsky performance score $>70$; (4) adequate organ function (white blood cell $>4.0 \times 10 / \mathrm{L}$; neutrophil $>2.0 \times 10 / \mathrm{L}$; hemoglobin $>90 \mathrm{~g} / \mathrm{L}$; platelet $>100 \times 10^{9} / \mathrm{L}$; aspartate aminotransferase/alanine transaminase $<2.5$ upper limit of normal; $\mathrm{Ccr}>60 \mathrm{~mL} / \mathrm{min}$ ); and (5) absence of pregnancy, lactation, and other malignant disease. The flow chart is described in Fig. 1. Routine evaluations were performed on patients, including physical examination, electrocardiography, head and neck magnetic resonance imaging (MRI) with contrast, nasopharyngoscopy and biopsy, chest and abdominal computed tomography (CT) with contrast and bone scan. The positron emission tomography computed tomography (PET-CT) was considered as an alternative to whole body examination. Whether patients have initial bone metastasis were based on the image diagnostic report before treatment. Bone metastasis was taken into consideration when either emission computed tomography or PET-CT showed evidence of bone lesions. Besides, other examines or PET-CT could not find metastasis in other organs. These patients were defined as the initial bone-only metastasis patients. Two radiologists determined all image diagnostic reports on the basis of the imaging diagnosis criteria. Plasma EBV DNA measurement was routinely performed and further examinations were considered when necessary.

\section{Chemotherapy and radiation therapy}

All eligible patients received the cisplatin-based combination PCT. Common PCT regimens included TPF (docetaxel [60 $\mathrm{mg} / \mathrm{m}^{2}$ day 1] combined with cisplatin [60 $\mathrm{mg} / \mathrm{m}^{2}$ day 1] plus 5-fluorouracil [500-800 mg/ $\mathrm{m}^{2}, 120$ hours]), PF (cisplatin [20-25 mg/ $\mathrm{m}^{2}$ days 1-3] combined with 5-fluorouracil [800$1,000 \mathrm{mg} / \mathrm{m}^{2}, 120$ hours $]$ ), and TP (docetaxel $\left[75 \mathrm{mg} / \mathrm{m}^{2}\right.$, day 1] combined with cisplatin [20-25 mg/ $\mathrm{m}^{2}$, days 1-3]). Chemotherapy was administered intravenously every 3 weeks. After PCT, 157 patients and 68 patients experienced LRRT and MRT respectively, using intensity modulated radiation therapy (IMRT) or two-dimensional conventional radiotherapy. The total dose of radiotherapy was 68-70 Gy for the nasopharynx and neck and 30-40 Gy for the metastatic site, 5 times a week, at approximately 2 Gy per fraction. The detailed design of the IMRT plan was generated using previous studies [13].

\section{Outcome and follow-up}

The primary endpoint of this study was overall survival (OS), which was defined as the time from the date of diag-
Table 1. Patient characteristics in our cohort

\begin{tabular}{|c|c|}
\hline Characteristic & No. $(\%)$ \\
\hline \multicolumn{2}{|l|}{ Age $(y r)$} \\
\hline \multicolumn{2}{|l|}{ Median (range) } \\
\hline$\leq 47$ & $114(50.4)$ \\
\hline$>47$ & $112(49.6)$ \\
\hline \multicolumn{2}{|l|}{ Sex } \\
\hline Male & $188(83.2)$ \\
\hline Female & $38(16.8)$ \\
\hline \multicolumn{2}{|c|}{ No. of metastatic lesions } \\
\hline$\leq 3$ & $132(58.4)$ \\
\hline$>3$ & $94(41.6)$ \\
\hline \multicolumn{2}{|l|}{ T categorya) } \\
\hline T1 & $11(4.9)$ \\
\hline T2 & $31(13.7)$ \\
\hline T3 & $111(49.1)$ \\
\hline $\mathrm{T} 4$ & $73(32.3)$ \\
\hline \multicolumn{2}{|l|}{$\mathrm{N}$ categorya) } \\
\hline N0 & $7(3.1)$ \\
\hline N1 & $42(18.6)$ \\
\hline N2 & $97(42.9)$ \\
\hline N3 & $80(35.4)$ \\
\hline \multicolumn{2}{|l|}{ PCT regimen } \\
\hline TPF & $64(28.3)$ \\
\hline TP & $60(26.5)$ \\
\hline PF & $56(24.8)$ \\
\hline Other regimens & $46(20.4)$ \\
\hline \multicolumn{2}{|c|}{ Pre-EBV DNA (copy/mL) } \\
\hline$<25,000$ & $113(50.0)$ \\
\hline$\geq 25,000$ & $113(50.0)$ \\
\hline \multicolumn{2}{|c|}{ EBV DNA after PCT } \\
\hline Undetectable $^{\mathrm{b})}$ & $128(56.6)$ \\
\hline 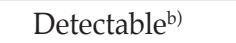 & $98(43.4)$ \\
\hline \multicolumn{2}{|l|}{ LRRT } \\
\hline Yes & $157(69.5)$ \\
\hline No & $69(30.5)$ \\
\hline \multicolumn{2}{|l|}{ MRT } \\
\hline Yes & $68(30.1)$ \\
\hline No & $158(69.9)$ \\
\hline
\end{tabular}

PCT, palliative chemotherapy; EBV, Epstein-Barr virus; TPF, cisplatin plus docetaxel plus 5-fluorouracil; TP, cisplatin plus docetaxel; PF, cisplatin plus 5-fluorouracil; LRRT, locoregional radiotherapy; MRT, metastasis radio-

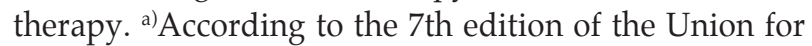
International Cancer Control/American Joint Committee

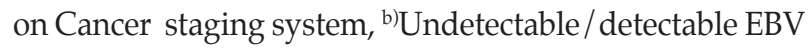
DNA levels after neoadjuvant chemotherapy are based on a cut-off value of 0 copy $/ \mathrm{mL}$. 
Table 2. Univariable analysis and multivariable analysis for overall survival in our cohort

\begin{tabular}{|c|c|c|c|c|}
\hline \multirow{2}{*}{ Characteristic } & \multicolumn{2}{|c|}{ Univariable analyses } & \multicolumn{2}{|c|}{ Multivariable analyses } \\
\hline & Hazard ratio $(95 \% \mathrm{CI})$ & p-value & Hazard ratio $(95 \% \mathrm{CI})$ & p-value \\
\hline \multicolumn{5}{|l|}{ Age (yr) } \\
\hline$\leq 47$ & Reference & & Reference & \\
\hline$>47$ & $1.07(0.73-1.56)$ & 0.728 & $0.86(0.58-1.29)$ & 0.467 \\
\hline \multicolumn{5}{|l|}{ Sex } \\
\hline Male & Reference & & Reference & \\
\hline Female & $1.05(0.63-1.745)$ & 0.846 & $1.30(0.77-2.19)$ & 0.336 \\
\hline \multicolumn{5}{|c|}{ No. of metastatic lesions } \\
\hline$\leq 3$ & Reference & & Reference & \\
\hline$>3$ & $2.53(1.72-3.72)$ & $<0.001$ & $1.92(1.26-2.94)$ & 0.003 \\
\hline \multicolumn{5}{|l|}{ T categorya) } \\
\hline T1-2 & Reference & & Reference & \\
\hline T3-4 & $1.01(0.62-1.65)$ & 0.964 & $1.05(0.63-1.73)$ & 0.857 \\
\hline \multicolumn{5}{|l|}{$\mathrm{N}$ category ${ }^{\mathrm{a})}$} \\
\hline N0-1 & Reference & & Reference & \\
\hline $\mathrm{N} 2-3$ & $1.97(1.19-3.28)$ & 0.009 & $1.69(0.99-2.90)$ & 0.054 \\
\hline \multicolumn{5}{|l|}{ PCT regimen } \\
\hline TPF & Reference & & Reference & \\
\hline TP & $1.05(0.61-1.81)$ & 0.850 & $1.07(0.61-1.88)$ & 0.819 \\
\hline PF & $1.23(0.73-2.06)$ & 0.433 & $1.39(0.80-2.41)$ & 0.242 \\
\hline Other regimens & $1.41(0.82-2.43)$ & 0.218 & $1.61(0.91-2.84)$ & 0.104 \\
\hline \multicolumn{5}{|c|}{ Pre-treatment EBV DNA (copy/mL) } \\
\hline$<25,000$ & Reference & & Reference & \\
\hline$\geq 25,000$ & $1.75(1.19-2.58)$ & 0.005 & $1.14(0.73-1.79)$ & 0.567 \\
\hline \multicolumn{5}{|c|}{ EBV DNA after PCT } \\
\hline Undetectable $^{\mathrm{b})}$ & Reference & & Reference & \\
\hline Detectable $^{\mathrm{b})}$ & $2.79(1.90-4.11)$ & $<0.001$ & $1.91(1.23-2.96)$ & 0.004 \\
\hline \multicolumn{5}{|l|}{ LRRT } \\
\hline No & Reference & & Reference & \\
\hline Yes & $0.39(0.27-0.59)$ & $<0.001$ & $0.48(0.31-0.75)$ & 0.001 \\
\hline \multicolumn{5}{|l|}{ MRT } \\
\hline No & Reference & & Reference & \\
\hline Yes & $0.77(0.50-1.19)$ & 0.243 & $1.21(0.76-1.91)$ & 0.422 \\
\hline
\end{tabular}

CI, confidence interval; PCT, palliative chemotherapy; TPF, cisplatin plus docetaxel plus 5-fluorouracil; TP, cisplatin plus docetaxel; PF, cisplatin plus 5-fluorouracil; EBV, Epstein-Barr virus; LRRT, locoregional radiotherapy; MRT, metastasis

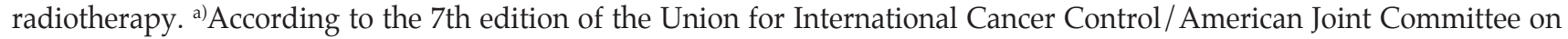

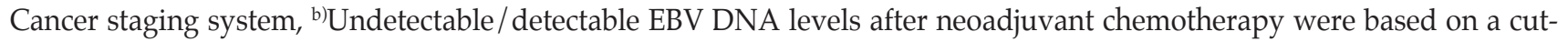
off value of 0 copy $/ \mathrm{mL}$, a Cox proportional hazard model was used to perform multivariate analyses.

nosis to the date of death of any cause. Patients were inspected every 3 months in the first 3 years and every 6 months thereafter until death. A series of evaluations were conducted including physical examination, nasopharyngoscopy, MRI with contrast of head and neck, CT/magnetic resonance with contrast of the metastatic sites, abdominal sonography, chest radiography and plasma EBV DNA measurement. PET-CT was considered when necessary.

\section{Statistical analyses}

Time-to-event data in different subgroups were analyzed using Kaplan-Meier curves and compared with the log-rank test. Univariable and multivariable Cox regression analyses were used to estimate the hazard ratios (HRs) and 95\% confidence intervals (CIs) for the relationship between the characteristic and overall survival. Statistical analyses were performed using the SPSS (Mac ver. 21.0, IBM Corp., Armonk, 
A

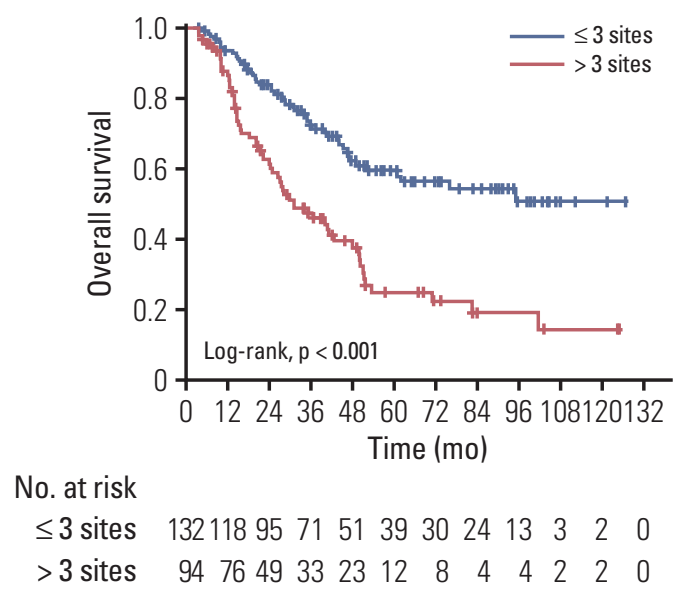

C

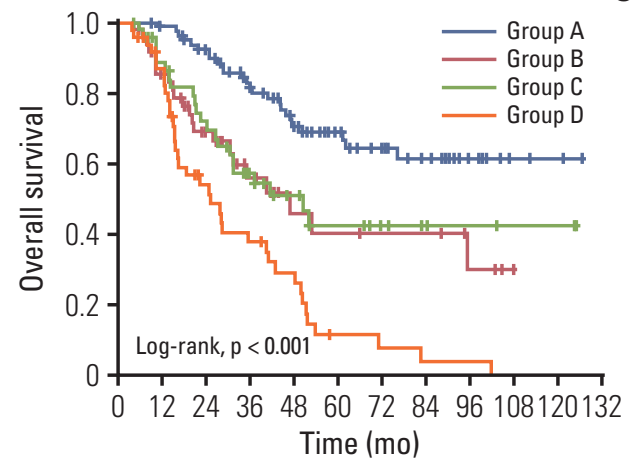

No. at risk

Group A $827970 \begin{array}{lllllllll}56 & 43 & 32 & 24 & 18 & 10 & 3 & 2 & 0\end{array}$

Group B $\quad \begin{array}{llllllllllll}50 & 39 & 25 & 15 & 8 & 7 & 6 & 6 & 3 & 0 & 0 & 0\end{array}$

Group C $46 \begin{array}{lllllllllll}38 & 29 & 19 & 13 & 9 & 6 & 3 & 3 & 2 & 2 & 0\end{array}$

Group D $48 \begin{array}{lllllllllll}38 & 20 & 14 & 10 & 3 & 2 & 1 & 1 & 0 & 0 & 0\end{array}$
A

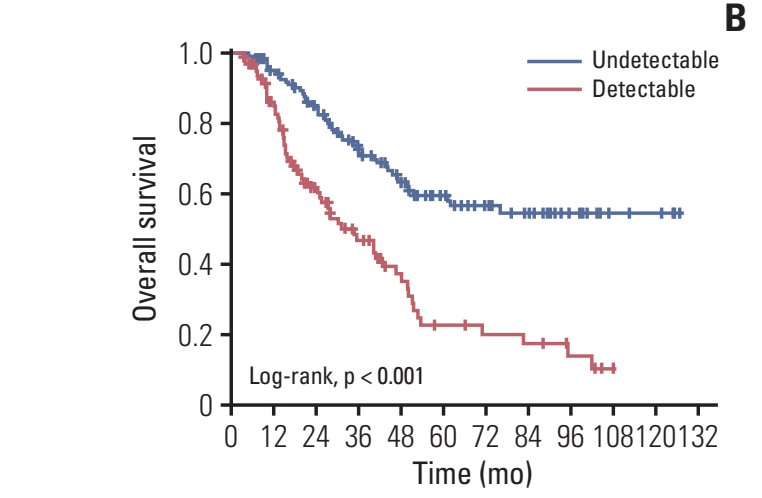

No. at risk

Undetectable 128117997556413021135440

Detectable $\quad 98 \quad 7745 \quad 2918 \quad 10 \quad 8 \quad 7 \quad 4 \quad 0 \quad 00$

Fig. 2. (A) Kaplan-Meier overall survival curves in 226 patients with bone-only metastatic nasopharyngeal carcinoma. Patients grouped by the number of metastatic lesions by Epstein-Barr virus (EBV) DNA after palliative chemotherapy (PCT) (B); by combination of lesion numbers and EBV DNA after PCT (C) and by risk stratification (D).

NY). All statistical tests in our study were 2-tailed. $p<0.05$ was considered to represent statistical significance.

\section{Ethical statement}

At our center, all patients signed informed consent prior to treatment, including their consent to treatment and clinical information for further prognostic analysis. This study was approved by the Research Ethics Committee of the Sun Yatsen University Cancer Center, China.

\section{Results}

\section{Patient characteristics and OS}

Among all 226 patients, the median age was 47 years (range, 18 to 74 years). Patients in our cohort were from NPC endemic areas with a male predominance $(83.2 \%)$. All patients underwent first-line PCT. After PCT, 157 patients (69.5\%) underwent LRRT and 69 patients $(30.1 \%)$ underwent MRT. With respect to number of lesions, 94 patients (41.6\%) had more than three metastatic lesions. EBV DNA was detected in 207 patients $(91.6 \%$ ) before PCT and only 98 patients $(43.4 \%)$ had detectable EBV DNA levels after PCT. Other 
Table 3. Survival rate and multivariable analysis for overall survival of patients in different risk groups

\begin{tabular}{lccccrr}
\multirow{2}{*}{ Risk group } & \multicolumn{2}{c}{ Survival rate } & & \multicolumn{3}{c}{ Multivariable analyses } \\
\cline { 2 - 3 } & 3-Year & 5-Year & & Adjusted HR & 95\% CI & p-value \\
Low-risk & 80.0 & 66.7 & Reference & & \\
Intermediate-risk & 54.9 & 41.3 & 2.24 & $1.33-3.75$ & 0.002 \\
High-risk & 37.8 & 11.6 & 3.69 & $2.01-6.79$ & $<0.001$ \\
\hline
\end{tabular}

Survival rate was calculated from Kaplan-Meier curve. Multivariable Cox regression model adjusted for age, sex, T category, $\mathrm{N}$ category, and pre-treatment Epstein-Barr virus DNA; palliative chemotherapy regimen, locoregional radiotherapy, and metastasis radiotherapy. CI, confidence interval.

characteristics of the 226 patients are listed in Table 1 . The median follow-up time was 33.9 months (range, 3 to 126 months). During follow-up, 105 patients died of tumor progression and two patients died of treatment-related toxicities.

\section{Univariable analysis and multivariable analysis}

All potential prognostic factors were considered in the Cox proportional hazards model. The cut-off value of the number of metastatic lesions for patient outcome was determined by receiver operating characteristic (ROC) curve analysis (3 metastasis lesions). The pre-treatment EBV DNA level was categorized based on the median value $(25,000$ copies $/ \mathrm{mL})$. The EBV DNA after PCT was scored on the basis of a detectable/ undetectable scale (a cut-off value of 0 copy $/ \mathrm{mL}$ ). Univariate analysis showed that the number of metastatic lesions, $\mathrm{N}$ category, pre-treatment EBV DNA, EBV DNA post-PCT, and LRRT were significantly associated with OS among patients with bone metastasis at primary diagnosis. In contrast, only the number of lesions, EBV DNA post-PCT, and LRRT remained significant in the multivariate analysis (Table 2). The Kaplan-Meier survival curves in patients are shown in Fig. 2A and B.

\section{Risk stratification}

According to the independent prognostic factors, we subdivided all patients into four subgroups: group A, three or fewer metastatic lesions and undetectable EBV DNA after PCT; group $B$, more than three metastatic lesions and undetectable EBV DNA after PCT; group C, three or fewer metastatic lesions and detectable EBV DNA after PCT; and group D, more than three metastatic lesions and detectable EBV DNA after PCT. Further pair-wise comparisons showed that group $B$ and group $C$ were not significantly different $(\mathrm{p}=$ 0.764 ), and that they both had significantly lower survival rates than group $\mathrm{A}$. They also had a higher survival rate than group D (S1 Table). Thus, we combined group B and group $\mathrm{C}$ as the intermediate-risk subgroup, with groups $\mathrm{A}$ and D serving as the low-risk and high-risk groups, respectively. Kaplan-Meier survival curves are shown in Fig. 2C and D. The survival rate and multivariate analysis for patients in different risk groups are listed in Table 3.

\section{Treatment outcome for those in different risk levels}

We further explored the impact of combined LRRT or MRT among patients with bone-only metastatic NPC in different risk groups. In the low- and intermediate-risk groups, the OS rate was significantly higher in patients treated with LRRT ( $p=0.006$ and $p=0.005$, respectively), while there was no survival benefit observed in high-risk patients $(\mathrm{p}=0.918)$ (Fig. 3A-C). Furthermore, patients in any risk group received no benefit from MRT (Fig. 3D-F). Then, we conducted stratified multivariate analysis for the different risk groups. HR was adjusted for age, sex, and other confounding variables. Fig. 4 showed the six groups of patients stratified by treatment modality and risk groups. Adjusted HR for OS increased from those in low-, intermediate-, and high-risk groups in all treatment modalities. LRRT was an independent prognostic factor in the low- and intermediate-risk groups ( $\mathrm{HR}_{\text {low, }}$ $0.23 ; 95 \% \mathrm{CI}, 0.09$ to $0.61 ; \mathrm{p}=0.003 ; \mathrm{HR}_{\text {intermediate, }} 0.40 ; 95 \% \mathrm{CI}$, 0.21 to $0.76 ; \mathrm{p}=0.005)$. However, MRT was not associated with a better OS in all groups of patients (Table 4).

\section{Discussion}

Bone is the most frequently involved organ in metastatic NPC patients [7-9]. In this study, we included 226 NPC patients with bone metastasis at primary diagnosis. This was based on the large cohort of bone-only metastasis NPC patients from endemic areas. OS among these patients ranged from 3 to 126 months, which indicates that long-term survival is possible in these patients. Therefore, it is necessary to find reliable prognostic factors that can be used to predict 
A

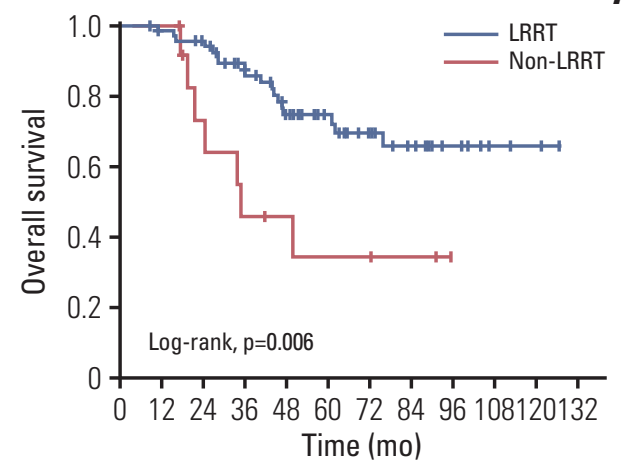

No. at risk

LRRT $6966 \quad 62513929211610 \quad 3 \quad 2 \quad 0$ Non-LRRT $11313 \quad 8 \quad 5 \quad 4 \quad 3 \quad 3 \quad 2 \quad 00000$

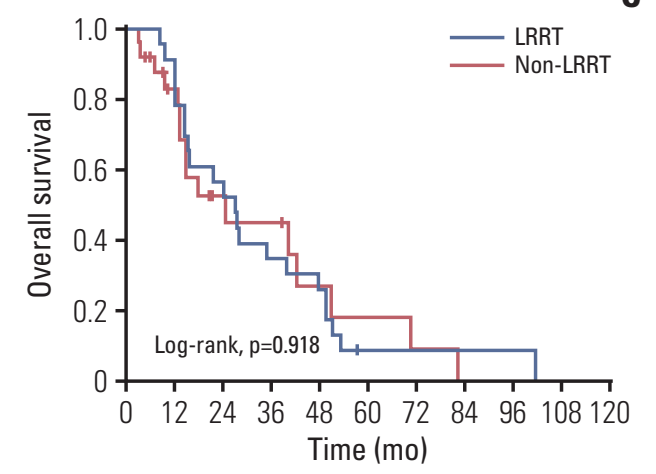

No. at risk

$\begin{array}{llllllllllll}\text { LRRT } & 23 & 21 & 13 & 8 & 7 & 1 & 1 & 1 & 1 & 0 & 0\end{array}$ $\begin{array}{llllllllllll}\text { Non-LRRT } & 25 & 17 & 7 & 6 & 3 & 2 & 1 & 0 & 0 & 0 & 0\end{array}$

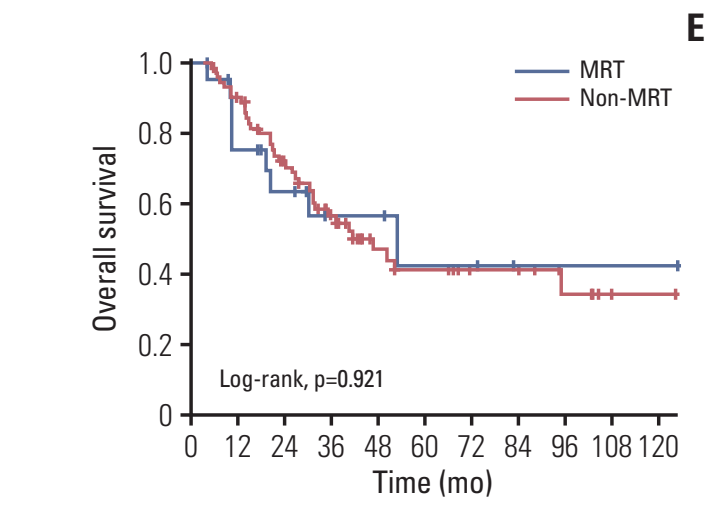

No. at risk

$\begin{array}{llllllllllll}\text { MRT } & 22 & 15 & 11 & 6 & 5 & 3 & 3 & 1 & 1 & 1 & 1\end{array}$ $\begin{array}{llllllllllll}\text { Non-MRT } & 74 & 62 & 43 & 28 & 16 & 13 & 9 & 8 & 5 & 1 & 1\end{array}$

E

C

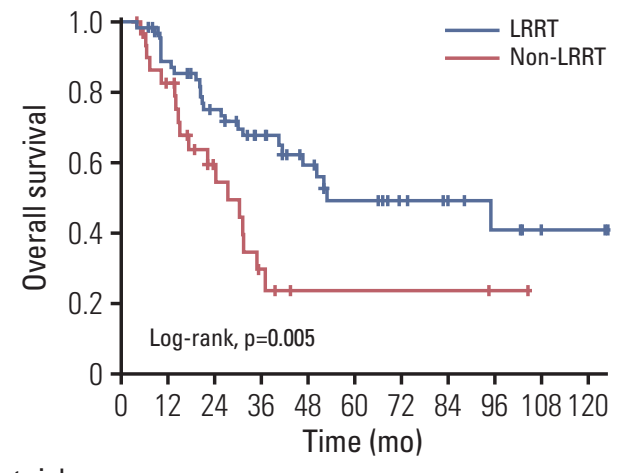

No. at risk

$\begin{array}{llllllllllll}\text { LRRT } & 65 & 54 & 43 & 29 & 19 & 14 & 10 & 7 & 5 & 2 & 2\end{array}$

$\begin{array}{llllllllllll}\text { Non-LRRT } & 31 & 23 & 11 & 5 & 2 & 2 & 2 & 2 & 1 & 0 & 0\end{array}$

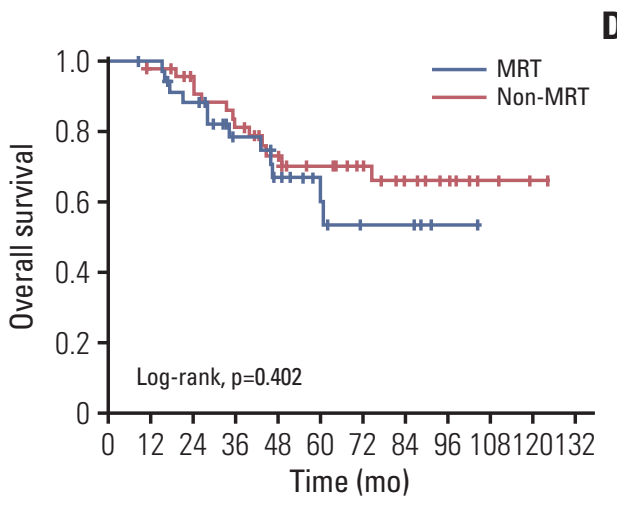

No. at risk

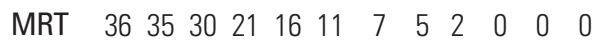

Non-MRT $46 \quad 44403527 \quad 21 \quad 17 \quad 13 \quad 8 \quad 3 \quad 2 \quad 0$

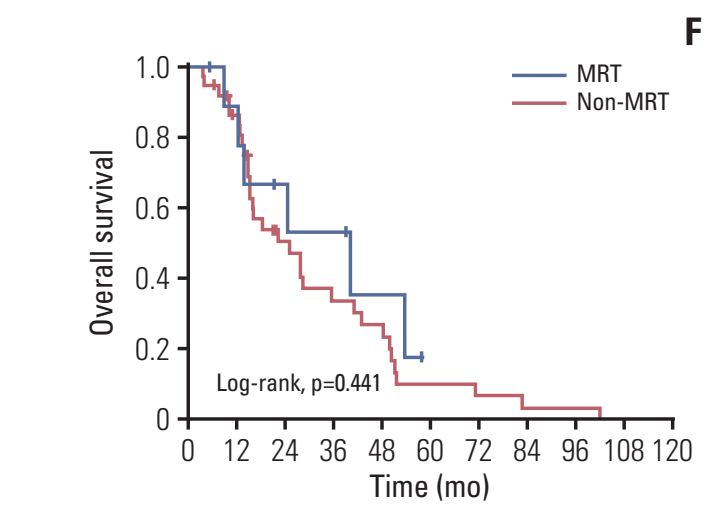

No. at risk

$\begin{array}{llllllllllll}\text { MRT } & 10 & 8 & 5 & 4 & 2 & 0 & 0 & 0 & 0 & 0 & 0\end{array}$

Non-MRT $38 \begin{array}{llllllllll}30 & 15 & 10 & 8 & 3 & 2 & 1 & 1 & 0 & 0\end{array}$

Fig. 3. Comparison of overall survival of patients in the locoregional radiotherapy (LRRT) and non-LRRT group: low-risk patients (A), intermediate-risk patients (B), and high-risk patients (C). Comparison of overall survival in patients in the metastasis radiotherapy (MRT) and non-MRT group: low-risk patients (D), intermediate-risk patients (E), and high-risk patients $(\mathrm{F})$. 
A
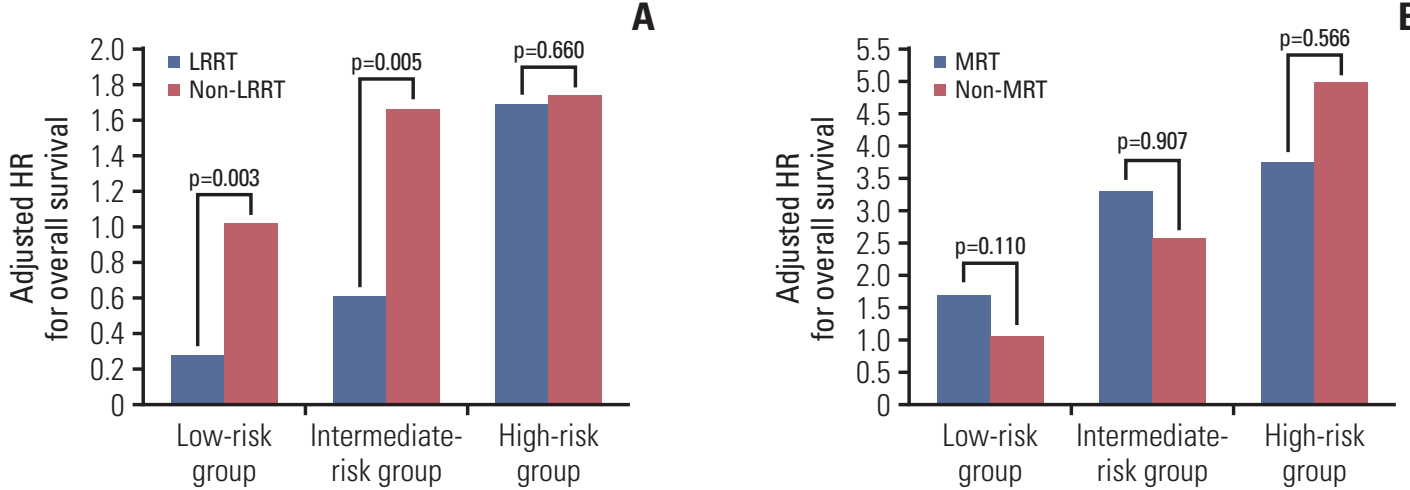

Fig. 4. Adjusted hazard ratios (HRs) for overall survival stratified by risk group and locoregional radiotherapy (LRRT) (A) or metastasis radiotherapy (MRT) (B) in patients with bone-only metastatic nasopharyngeal carcinoma. The low-risk group not combined with LRRT/MRT was the reference group $(\mathrm{HR}, 1)$. The adjusted variables were age, sex, T category, N category, and pre-treatment Epstein-Barr virus DNA.

Table 4. Multivariable analysis for overall survival by treatment modality in stratified risk groups

\begin{tabular}{|c|c|c|c|}
\hline \multirow{2}{*}{ Treatment modality } & \multicolumn{3}{|c|}{ Multivariable analyses } \\
\hline & $\overline{\text { Adjusted HR }}$ & $95 \% \mathrm{CI}$ & p-value \\
\hline \multicolumn{4}{|l|}{ LRRT (yes vs. no) } \\
\hline Low-risk & 0.23 & $0.09-0.61$ & 0.003 \\
\hline Intermediate-risk & 0.40 & $0.21-0.76$ & 0.005 \\
\hline High-risk & 0.85 & $0.41-1.76$ & 0.660 \\
\hline \multicolumn{4}{|l|}{ MRT (yes vs. no) } \\
\hline Low-risk & 1.98 & $0.54-2.90$ & 0.110 \\
\hline Intermediate-risk & 1.05 & $0.50-2.21$ & 0.907 \\
\hline High-risk & 0.76 & $0.29-1.95$ & 0.566 \\
\hline
\end{tabular}

Multivariable cox regression model adjusted for age, sex, T category, N category, and pre-treatment Epstein-Barr virus DNA.

$\mathrm{HR}$, hazard ratio; CI, confidence interval; LRRT, locoregional radiotherapy; MRT, metastasis radiotherapy.

survival more accurately, and guide appropriate treatment. Consequently, we explored the prognostic factors and the effect of treatment methods among them. There were several notable finding in our study. First, the number of metastatic lesions and EBV-DNA level are independent prognostic factors for OS in NPC patients with bone metastasis and these could be used as a basis for risk stratification. Secondly, LRRT was a valid therapeutic method, which should be combined with PCT in low- and intermediate-risk patients. Thirdly, MRT did not confer any survival benefit in any risk groups in our analysis. Thus, local treatment of distant metastasis should be carefully considered in clinical application.

EBV DNA, which is measured by real-time quantitative polymerase chain reaction, has been demonstrated to be closely associated with NPC [14]. In non-metastatic NPC patients, several studies have verified the prognostic value of EBV DNA levels before treatment (pre-EBV DNA) and EBV DNA levels after concurrent chemoradiation therapy/ radiotherapy (post-EBV DNA) [15,16]. In local advanced patients NPC who undergo induction chemotherapy (IC) before radiotherapy, our previously study showed that patients had undetectable EBV DNA after IC achieved higher progression-free survival and distant metastasis-free survival compared with patients who had detectable EBV DNA after IC [17]. Moreover, in metastatic/recurrent NPC patients, the predictive value of EBV for prognosis has also been demonstrated [18]. In our study, the plasma EBV DNA level was measured before treatment and after PCT in all patients. Univariate analysis showed that the EBV DNA level at these two time points was both associated with OS of NPC patients with bone-only metastasis, while detection of EBV DNA after PCT remained significant in multivariate analysis, indicating 
that undetectable EBV DNA after PCT was a better prognostic factor in these types of patients.

The number of metastatic lesions was closely related to the tumor burden. In metastatic NPC patients, several studies have demonstrated that patients with single metastasis achieve higher OS compared with patients with multiple metastases $[19,20]$. According to the results of studies assessing other types of malignancy, patients with multiple bone metastases also had poor clinical outcome [21-23]. However, few studies have focused on the prognostic value of the lesion number in survival among NPC patients with boneonly metastasis. In our study, the cut-off values of lesion number for OS was three based on the ROC analysis. We then considered this as a potential prognostic factor, which was included in univariate and multivariate analysis. It is intriguing that the number of lesions was an independent factor for OS in the multivariate analysis. Our result was in accordance with a previous study [5]. These findings suggest that more than three metastatic lesion could be used to establish risk stratification in bone-only metastatic NPC patients.

The treatment strategy for metastatic NPC patients remains a subject of great debate [24]. Platinum-based PCT is the most widely used treatment in these types of patients with objective response rates of 55\%-80\% [25,26]. Recently, several studies demonstrated that local control of primary tumor by radiotherapy could prolong survival $[4,19,27]$. Moreover, according to the NCCN guidelines, LRRT could benefit metastatic NPC patients with limited sites or with low tumor burden [12]. Thus, there still remains the issue of who will benefit from the combined treatment and further studies are required to resolve this. In the stratified analysis, we found that LRRT significantly improved OS in low- and intermediate-risk patients but conferred no benefit to high-risk patients. In accordance with the American College of Radiology therapeutic guidelines for bone metastasis, the management of patients with serious illnesses or conditions should concentrate on improving end-stage quality of life and maintaining neurological function [28]. In effect, aggressive treatment is not a suitable treatment for such individuals.

There is no consensus on whether local treatment of metastatic lesions is associated with a better OS in bone metastasis NPC patients due to the limited number of studies, which comprise small numbers of cases. In some case reports, radiotherapy of metastatic lesions has been observed to bring survival benefits for patients with bone-only oligometastatic lesions [29,30]. However, according to our results, all patients did not attain survival benefit from MRT, even for patients considered to be low risk. It has been suggested that better survival outcome in patients with bone-only oligometastatic lesions may be due to systemic PCT and LRRT, and not to MRT.

There were several limitations to our study. First, ours was a retrospective study so the evidence supporting these conclusions is not sufficient or convincing because of the potential for selective bias. Secondly, the cohort was obtained from an endemic area in one treatment center and may not be generally representative of patients with bone-only metastatic NPC. Finally, only 226 patients were involved in the study duo to the low incidence rate. For these reasons, a multiinstitutional prospective study is necessary to validate our results in the future.

\section{Electronic Supplementary Material}

Supplementary materials are available at Cancer Research and Treatment website (https: // www.e-crt.org).

\section{Conflicts of Interest}

Conflict of interest relevant to this article was not reported.

\section{References}

1. Torre LA, Bray F, Siegel RL, Ferlay J, Lortet-Tieulent J, Jemal A. Global cancer statistics, 2012. CA Cancer J Clin. 2015;65:87108.

2. Lee AW, Lin JC, Ng WT. Current management of nasopharyngeal cancer. Semin Radiat Oncol. 2012;22:233-44.

3. Tang LQ, Chen QY, Fan W, Liu H, Zhang L, Guo L, et al. Prospective study of tailoring whole-body dual-modality [18F]fluorodeoxyglucose positron emission tomography/ computed tomography with plasma Epstein-Barr virus DNA for detecting distant metastasis in endemic nasopharyngeal carcinoma at initial staging. J Clin Oncol. 2013;31:2861-9.

4. Chen MY, Jiang R, Guo L, Zou X, Liu Q, Sun R, et al. Locoregional radiotherapy in patients with distant metastases of nasopharyngeal carcinoma at diagnosis. Chin J Cancer. 2013; 32:604-13.

5. Shen L, Dong J, Li S, Wang Y, Dong A, Shu W, et al. M1 stage subdivision and treatment outcome of patients with bone-only metastasis of nasopharyngeal carcinoma. Oncologist. 2015;20: 291-8.

6. Pan CC, Lu J, Yu JR, Chen P, Li W, Huang ZL, et al. Challenges 
in the modification of the M1 stage of the TNM staging system for nasopharyngeal carcinoma: a study of 1027 cases and review of the literature. Exp Ther Med. 2012;4:334-8.

7. Ong YK, Heng DM, Chung B, Leong SS, Wee J, Fong KW, et al. Design of a prognostic index score for metastatic nasopharyngeal carcinoma. Eur J Cancer. 2003;39:1535-41.

8. Leung SF, Teo PM, Shiu WW, Tsao SY, Leung TW. Clinical features and management of distant metastases of nasopharyngeal carcinoma. J Otolaryngol. 1991;20:27-9.

9. Bensouda Y, Kaikani W, Ahbeddou N, Rahhali R, Jabri M, Mrabti $\mathrm{H}$, et al. Treatment for metastatic nasopharyngeal carcinoma. Eur Ann Otorhinolaryngol Head Neck Dis. 2011;128: 79-85.

10. Chen C, Wu JB, Jiang H, Gao J, Chen JX, Pan CC, et al. A prognostic score for nasopharyngeal Carcinoma with bone metastasis: development and validation from multicenter. J Cancer. 2018;9:797-806.

11. He S, Wang Y, Peng H, Yang L, Chen H, Liang S, et al. Pretreatment alkaline phosphatase and Epstein-Barr virus DNA predict poor prognosis and response to salvage radiotherapy in patients with nasopharyngeal carcinoma and metachronous bone-only metastasis. J Cancer. 2017;8:417-24.

12. Pfister DG, Ang KK, Brizel DM, Burtness BA, Busse PM, Caudell JJ, et al. Head and neck cancers, version 2.2013. Featured updates to the NCCN guidelines. J Natl Compr Canc Netw. 2013;11:917-23.

13. Sun Y, Yu XL, Luo W, Lee AW, Wee JT, Lee N, et al. Recommendation for a contouring method and atlas of organs at risk in nasopharyngeal carcinoma patients receiving intensitymodulated radiotherapy. Radiother Oncol. 2014;110:390-7.

14. Lo YM, Chan LY, Lo KW, Leung SF, Zhang J, Chan AT, et al. Quantitative analysis of cell-free Epstein-Barr virus DNA in plasma of patients with nasopharyngeal carcinoma. Cancer Res. 1999;59:1188-91.

15. Lin JC, Wang WY, Chen KY, Wei YH, Liang WM, Jan JS, et al. Quantification of plasma Epstein-Barr virus DNA in patients with advanced nasopharyngeal carcinoma. N Engl J Med. 2004;350:2461-70.

16. Chan AT, Lo YM, Zee B, Chan LY, Ma BB, Leung SF, et al. Plasma Epstein-Barr virus DNA and residual disease after radiotherapy for undifferentiated nasopharyngeal carcinoma. J Natl Cancer Inst. 2002;94:1614-9.

17. Liu LT, Tang LQ, Chen QY, Zhang L, Guo SS, Guo L, et al. The prognostic value of plasma Epstein-Barr viral DNA and tumor response to neoadjuvant chemotherapy in advanced-stage nasopharyngeal carcinoma. Int J Radiat Oncol Biol Phys. 2015;93:862-9.

18. An X, Wang FH, Ding PR, Deng L, Jiang WQ, Zhang L, et al. Plasma Epstein-Barr virus DNA level strongly predicts sur- vival in metastatic/recurrent nasopharyngeal carcinoma treated with palliative chemotherapy. Cancer. 2011;117:37507.

19. Zou X, You R, Liu H, He YX, Xie GF, Xie ZH, et al. Establishment and validation of M1 stage subdivisions for de novo metastatic nasopharyngeal carcinoma to better predict prognosis and guide treatment. Eur J Cancer. 2017;77:117-26.

20. Zeng L, Tian YM, Huang Y, Sun XM, Wang FH, Deng XW, et al. Retrospective analysis of 234 nasopharyngeal carcinoma patients with distant metastasis at initial diagnosis: therapeutic approaches and prognostic factors. PLoS One. 2014;9: e108070.

21. Bhandari V, Jain RK. A retrospective study of incidence of bone metastasis in head and neck cancer. J Cancer Res Ther. 2013;9:90-3.

22. Knudson G, Grinis G, Lopez-Majano V, Sansi P, Targonski P, Rubenstein $\mathrm{M}$, et al. Bone scan as a stratification variable in advanced prostate cancer. Cancer. 1991;68:316-20.

23. Yoon A, Choi CH, Kim TH, Choi JK, Park JY, Lee YY, et al. Bone metastasis in primary endometrial carcinoma: features, outcomes, and predictors. Int J Gynecol Cancer. 2014;24:10712.

24. Fandi A, Bachouchi M, Azli N, Taamma A, Boussen H, Wibault $\mathrm{P}$, et al. Long-term disease-free survivors in metastatic undifferentiated carcinoma of nasopharyngeal type. J Clin Oncol. 2000;18:1324-30.

25. Ji JH; Korean Cancer Study Group (KCSG), Yun T, Kim SB, Kang JH, Park JC, et al. A prospective multicentre phase II study of cisplatin and weekly docetaxel as first-line treatment for recurrent or metastatic nasopharyngeal cancer (KCSG HN07-01). Eur J Cancer. 2012;48:3198-204.

26. Chen C, Wang FH, An X, Luo HY, Wang ZQ, Liang Y, et al. Triplet combination with paclitaxel, cisplatin and 5-FU is effective in metastatic and/or recurrent nasopharyngeal carcinoma. Cancer Chemother Pharmacol. 2013;71:371-8.

27. Yeh SA, Tang Y, Lui CC, Huang EY. Treatment outcomes of patients with AJCC stage IVC nasopharyngeal carcinoma: benefits of primary radiotherapy. Jpn J Clin Oncol. 2006;36: 132-6.

28. Trilling GM, Cho H, Ugas MA, Saeed S, Katunda A, Jerjes W, et al. Spinal metastasis in head and neck cancer. Head Neck Oncol. 2012;4:36.

29. Khot A, Love C, Garg MK, Haigentz M Jr. Long-term disease control in a patient with recurrent bone-only oligometastatic nasopharyngeal carcinoma. J Clin Oncol. 2016;34:e25-6.

30. Lim A, Corry J, Lau E, Rischin D. Prolonged remission in a patient with nasopharyngeal carcinoma with a solitary bone metastasis. J Clin Oncol. 2011;29:e135-7. 